\title{
AN ILLUSTRATED BAR BAHLUL LEXICON
}

\section{(MS ST. GEORGE, ALQOSH; HMML DCA 84)}

Manuscripts provide room for the scribe to communicate directly with the reader through peritexts and illustrations. In a way, lexica are ideal for this as the scribe is free to add his own contribution between the letters of the alphabet. So does our scribe, Shammas "Deacon" 'İsā, who write this MS in AG 2186 (I875 CE). The scribe also gives the Islamic era of 1292 .

The lexicon begins with the three-sided border, common in many Syriac MSS, which is found on $\mathrm{f} .3^{\mathrm{v}}$. The scribe then adds decorative illustrations at the end of letter sections. These usually occupying part or most of a column. The illustration on $f$. $99^{\mathrm{v}}$, for example, is at the end of the letter $\supset$ and the beginning of the letter $\neg$. What is more interesting is the poetry, sometimes in Garshuni and sometimes in Arabic, which the scribe adds between letter sections. In this case, we have:

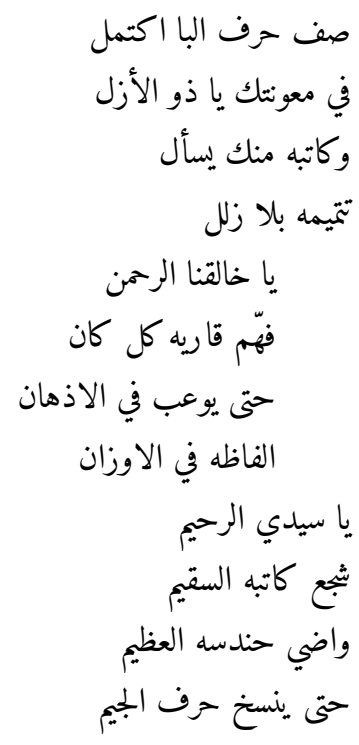


In the first stanza, the scribe addresses God, "the setting of the letter $b$ is completed with your help, O eternal one" and asks for "its completion without a mistake." The second stanza is devoted to the reader where the scribe asks "our merciful creator" to make the reader, whoever he was (i.e. of any scholastic level) comprehend the words in the lexicon. In the third stanza, the scribe then asks God to give him courage and "lighten the darkness" so he can finish the letter $j$ (whose name rhymes with the stanza).

The verse, while in Arabic, exhibits Syriac poetic structure that can only be understood if read in a quasi-colloquial manner. The meter seems to be the Syriac sevensyllabic one (the so-called Ephrem meter). One has to read it colloquially. One possible reading of the first stanza may be (this is my own reading and a local from the region may have different vowels):

saf harf el-bā 'ik-ta-mal

fì m-'un-tak yā dī al-'a-zal

wkä-tib-bu min-ka yas-'al

tat-mi-ma-bu bla za-lal

The scribe completed the manuscript for the user of two parishes in Alqosh, St. George and St. Michael (f. 489) ). 


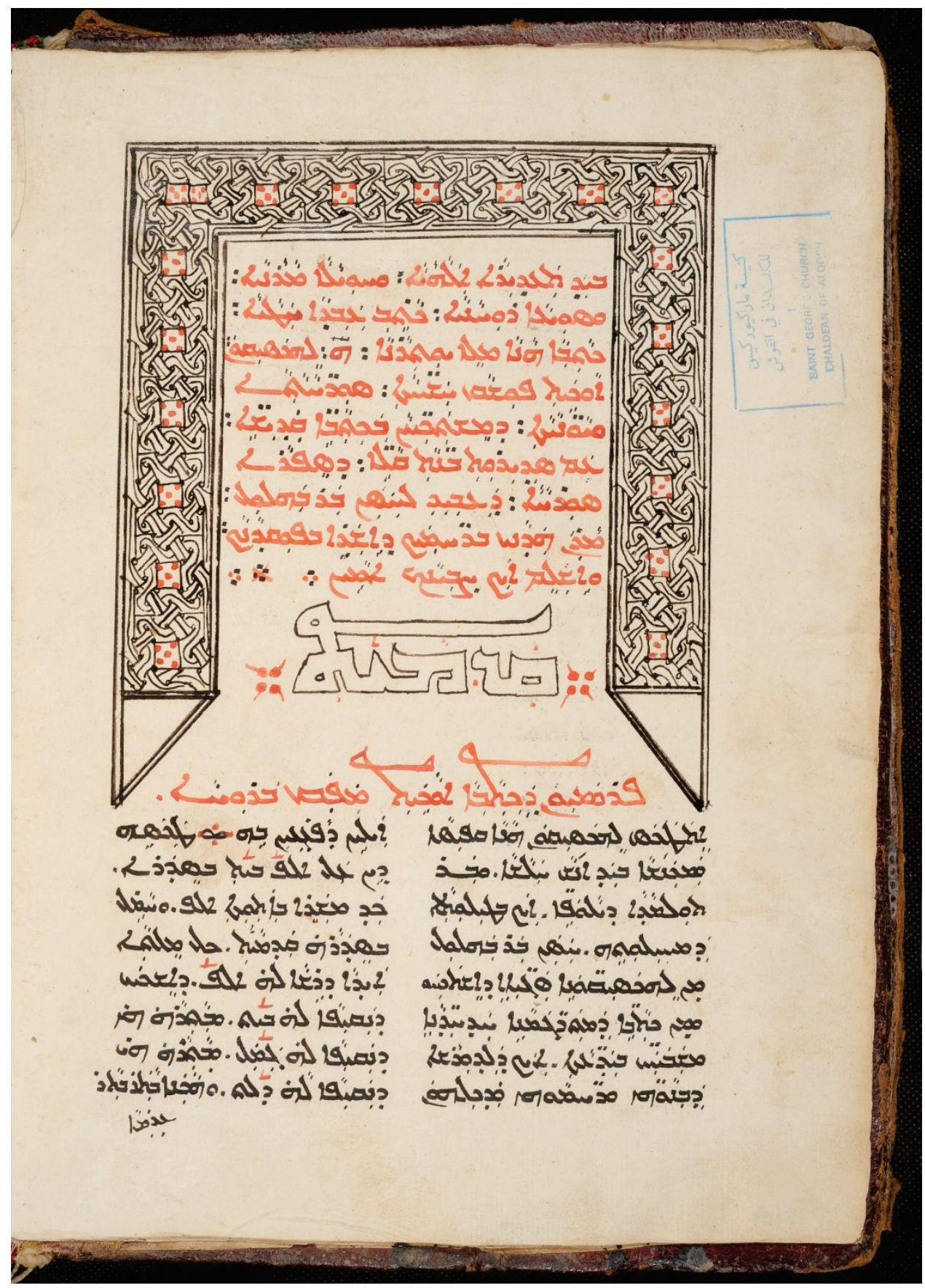

MS St. George, Alqosh; HMML DCA 84, folio 3v. 


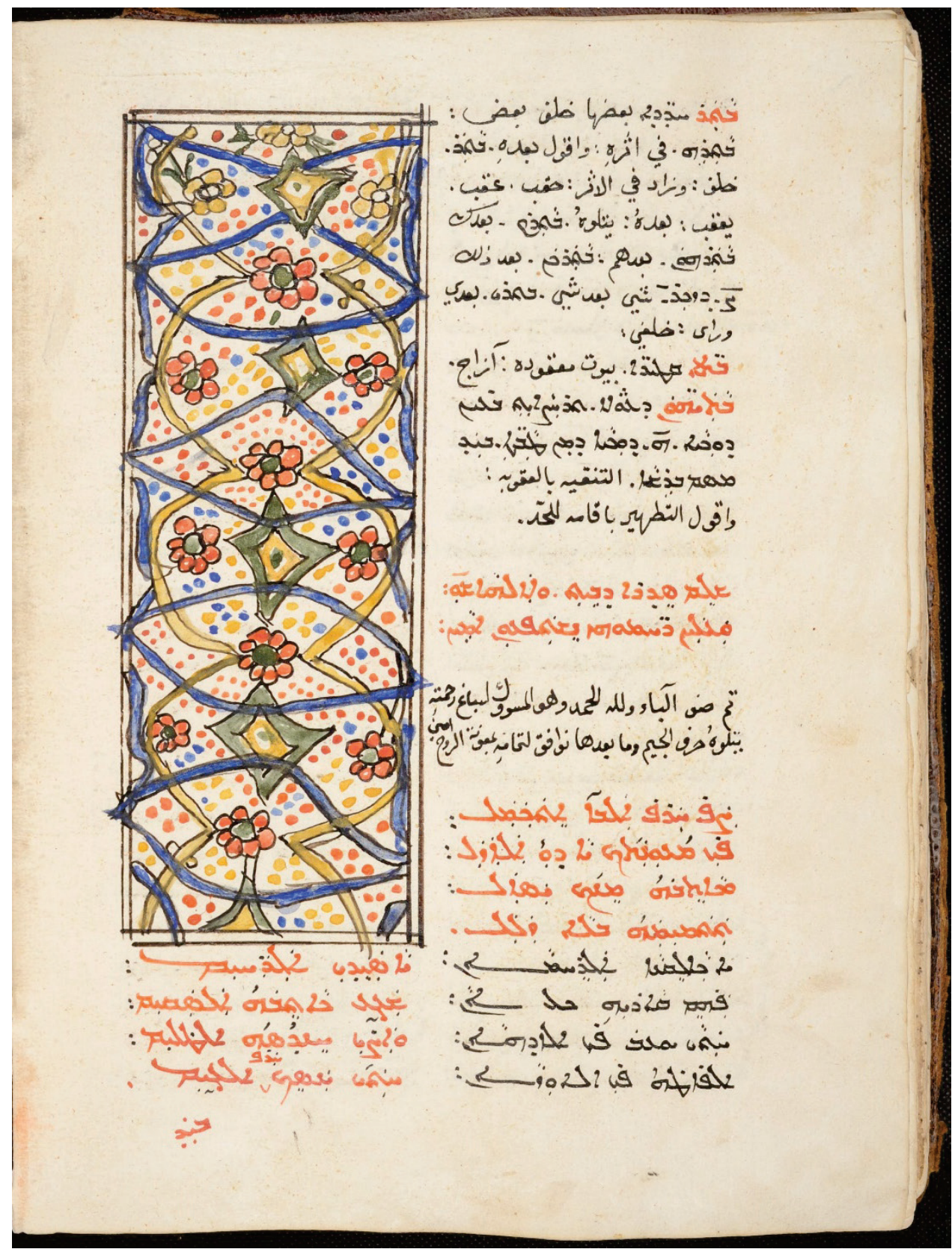

MS St. George, Alqosh; HMML DCA 84, folio 99v. 


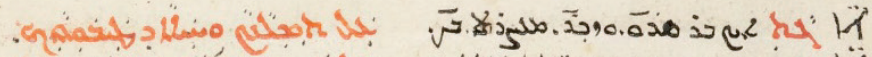
:

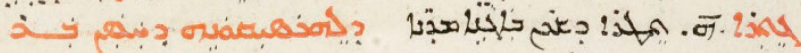

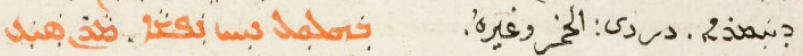
-

$$
\text { - }
$$

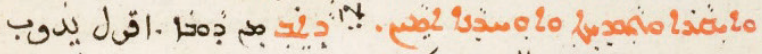

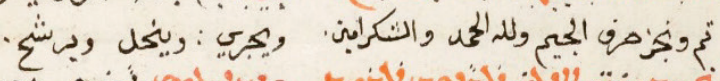

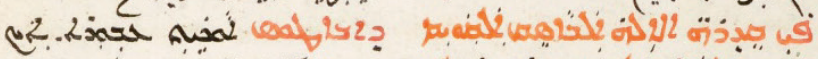

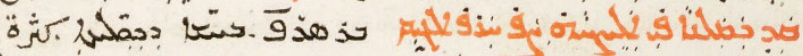

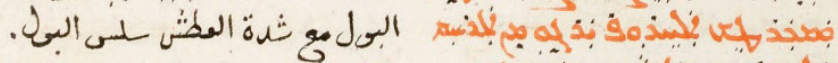

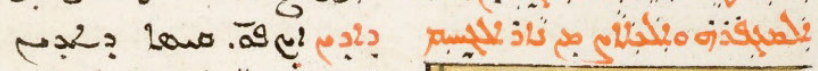

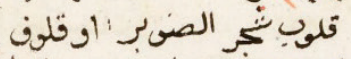

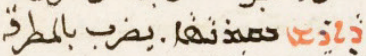

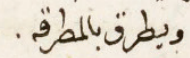

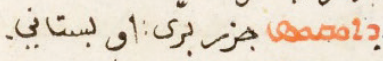

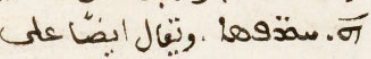

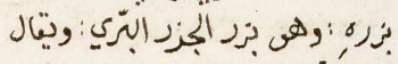

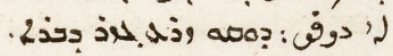

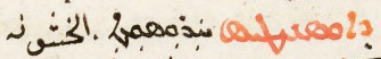

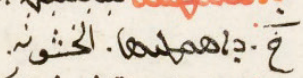

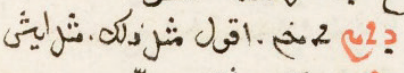

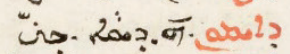
?

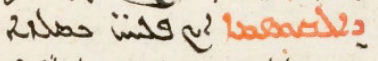

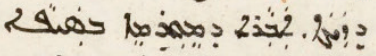
? :

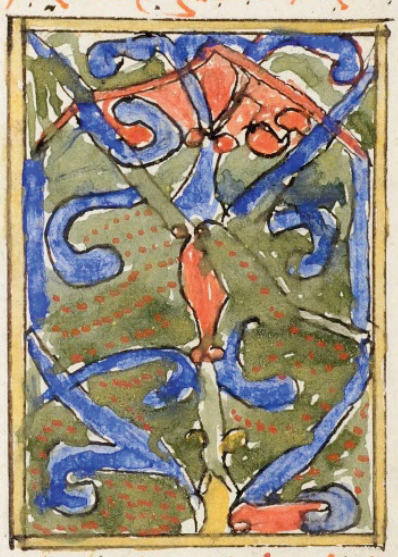

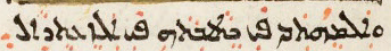

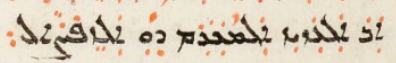

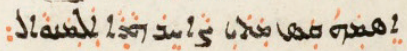
son?

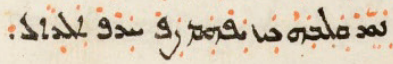

MS St. George, Alqosh; HMML DCA 84, folio II8v. 


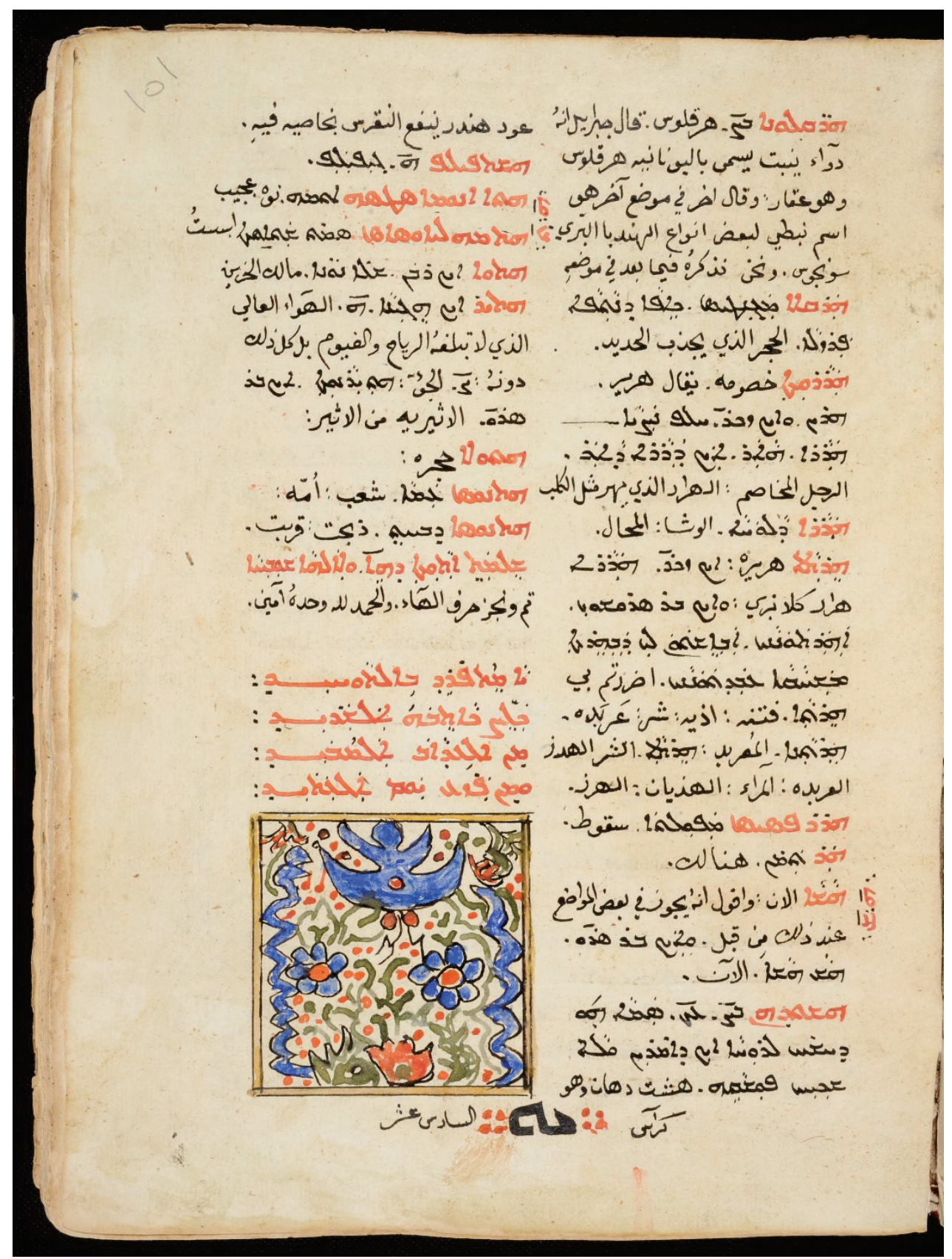

MS St. George, Alqosh; HMML DCA 84, folio I5Ir. 


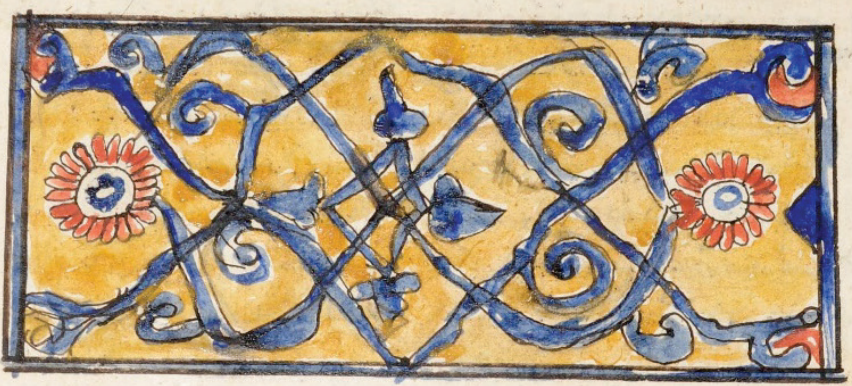

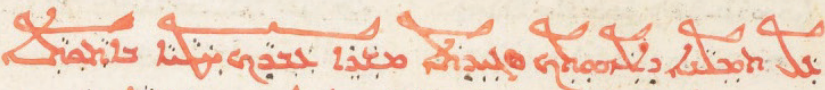

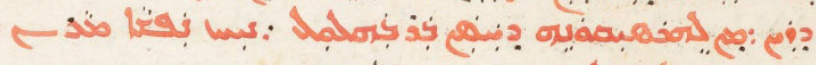
"

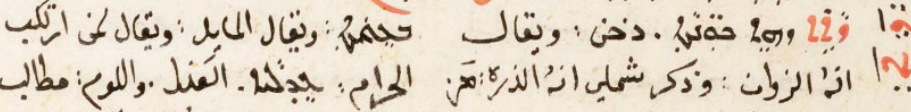

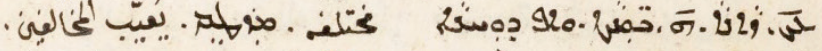

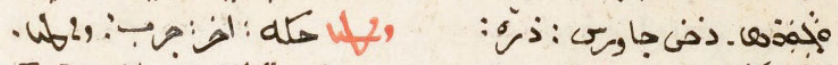

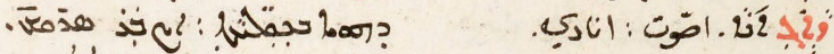

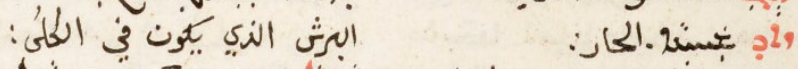

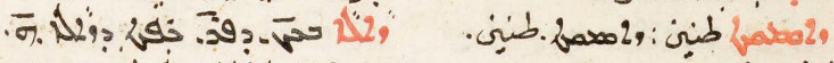

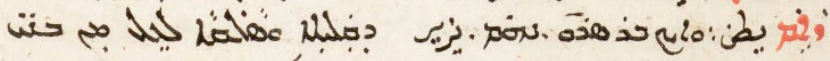

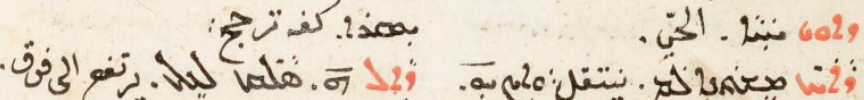
. 6029

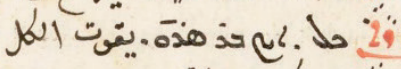

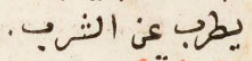

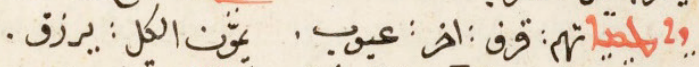

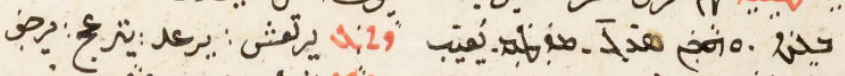

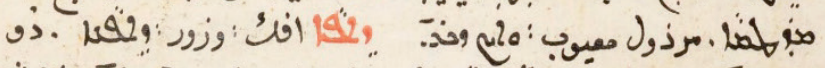

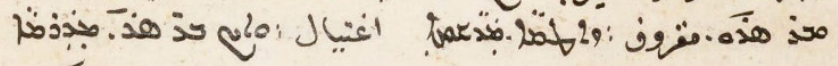

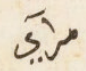

MS St. George, Alqosh; HMML DCA 84, folio I53v. 


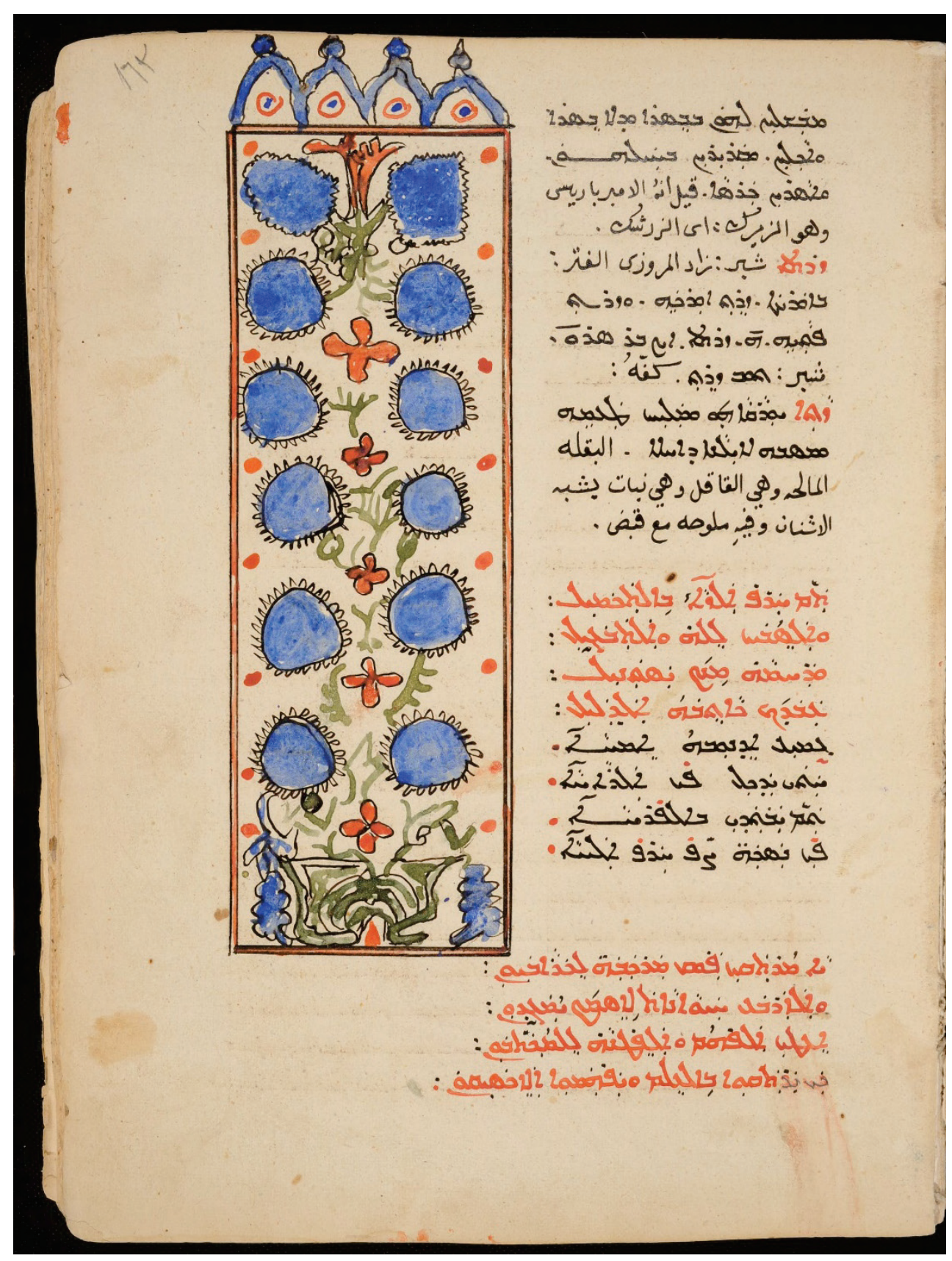

MS St. George, Alqosh; HMML DCA 84, folio I63r. 


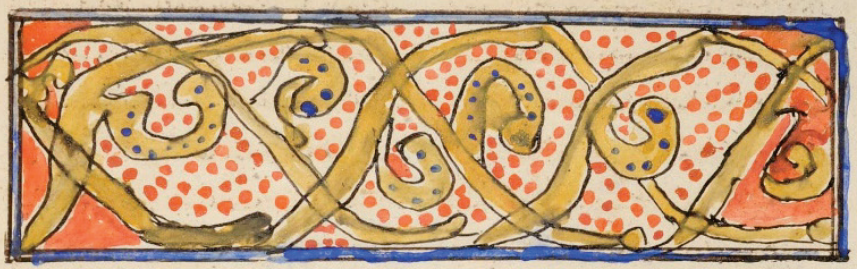

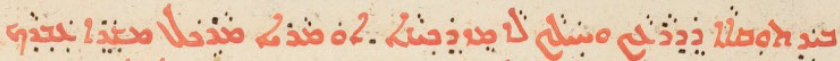

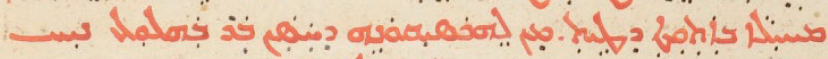

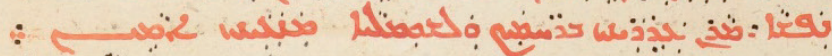

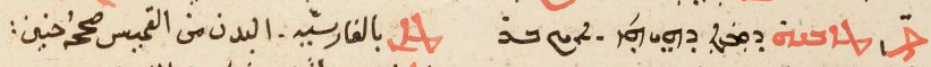

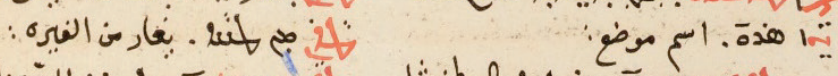

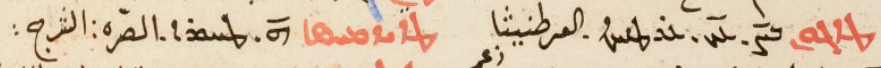
"5

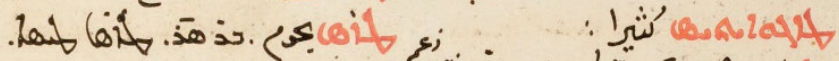

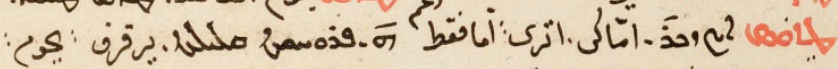

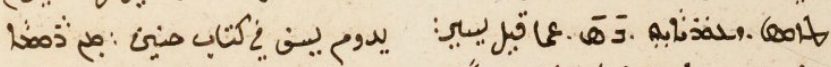

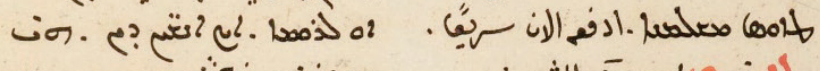

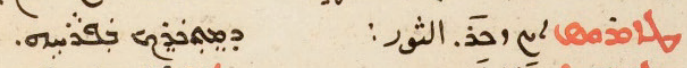

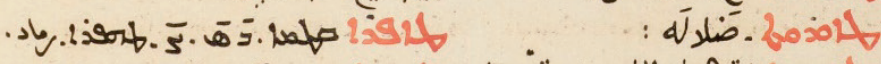

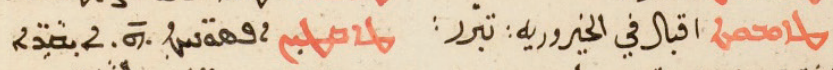

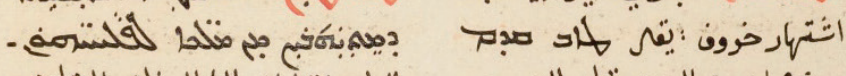

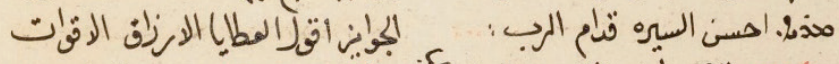

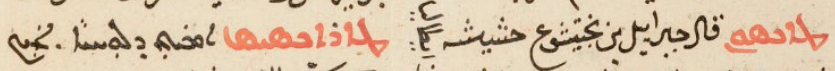

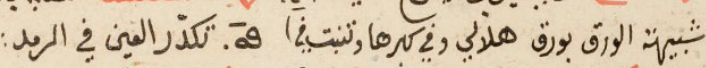

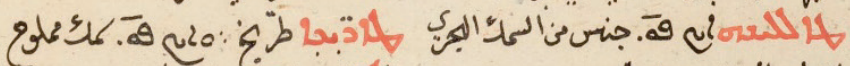

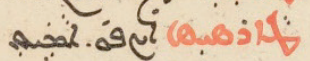

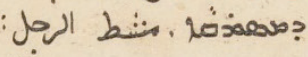
نهإ. iें :

MS St. George, Alqosh; HMML DCA 84, folio I84v. 


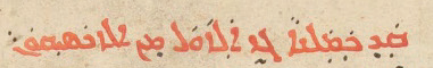
S

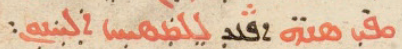

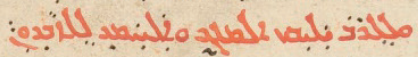

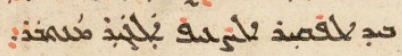

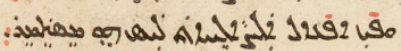

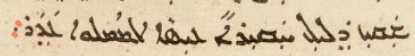

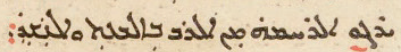

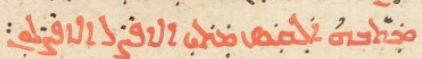

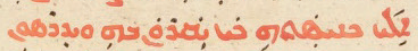

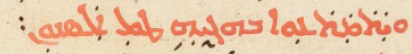

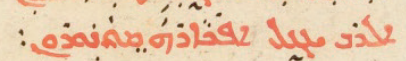

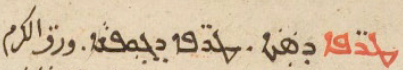
خذانها :

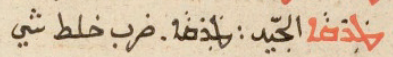

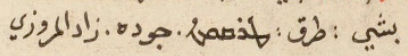

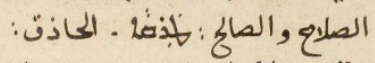

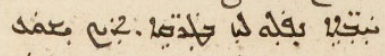

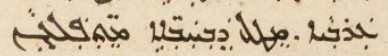
?

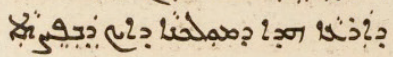

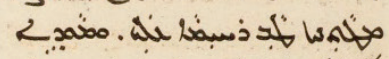

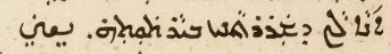

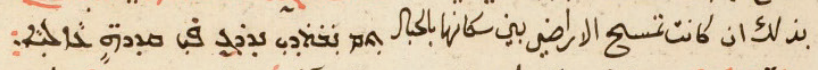

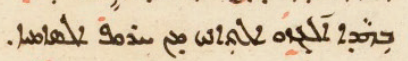

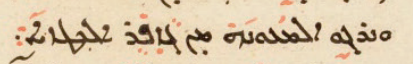
ها

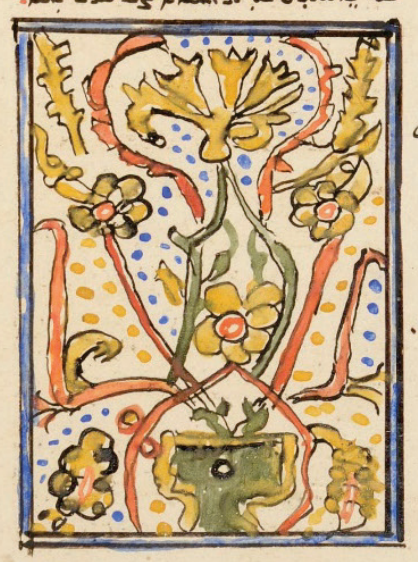

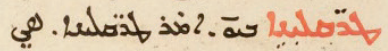

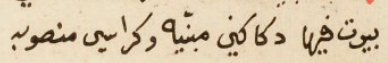

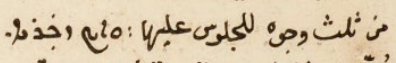

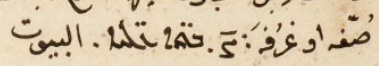
الفالير: المانت الهاليه:

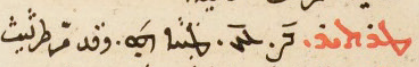

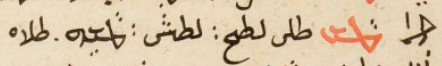

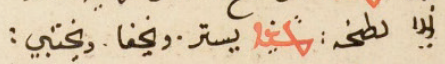

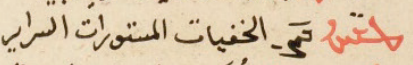

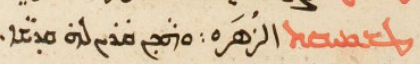

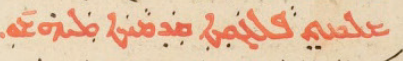

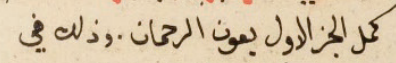

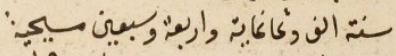

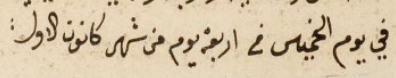

MS St. George, Alqosh; HMML DCA 84, folio 196v. 


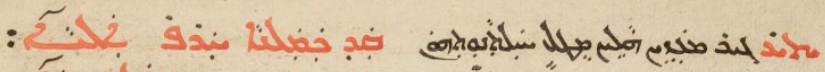

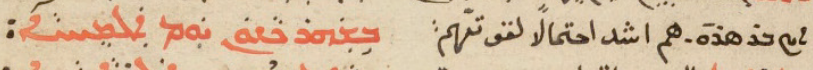

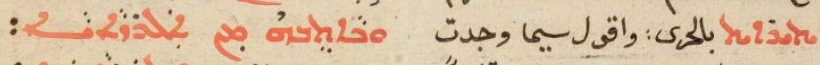

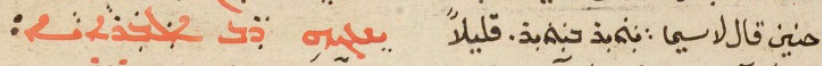

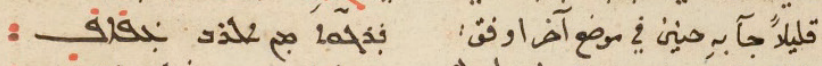

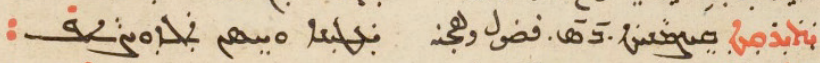

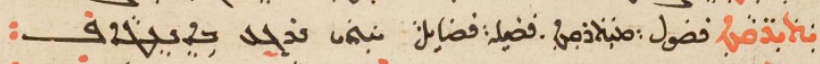

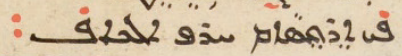

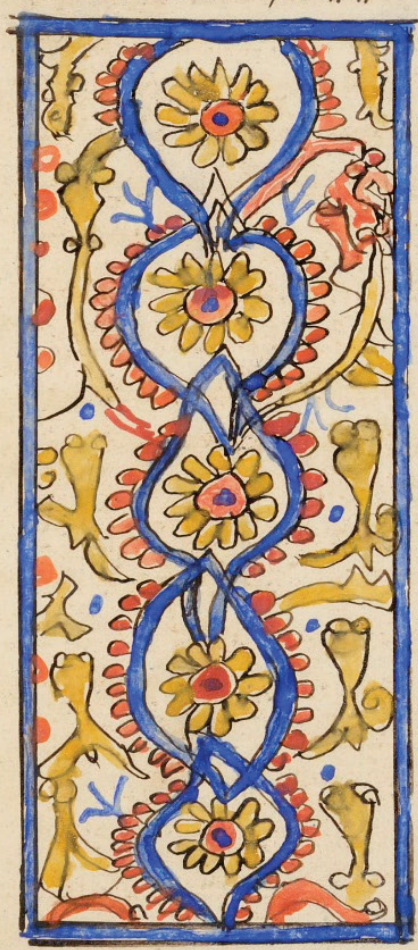

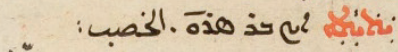

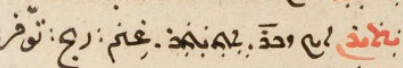
:

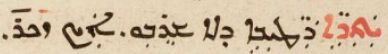

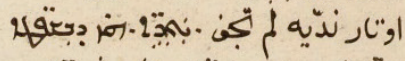

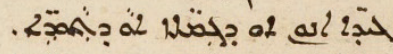

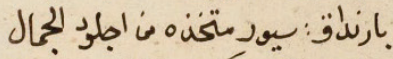

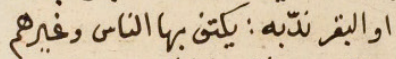

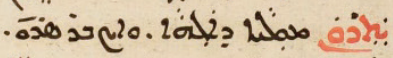

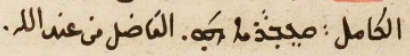

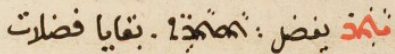

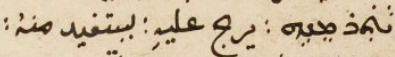
بهن .

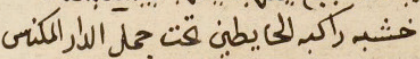

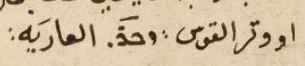
- ar 20)

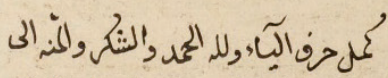

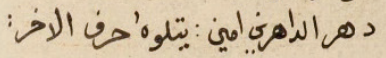

MS St. George, Alqosh; HMML DCA 84, folio 202v. 


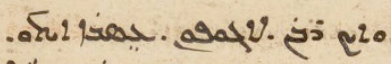

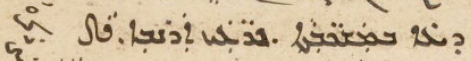

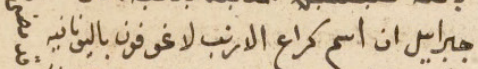

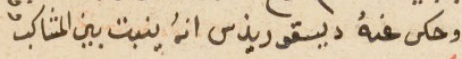
ل كئ

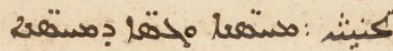

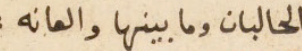

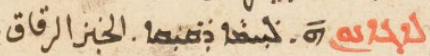
.

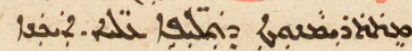

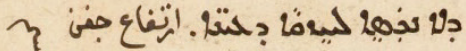

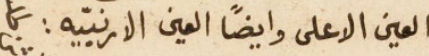

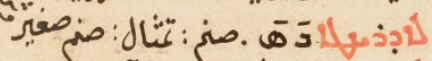

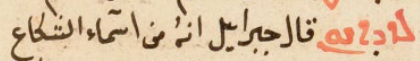

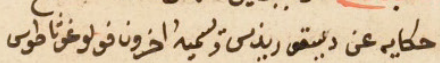

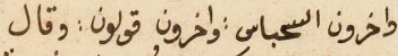

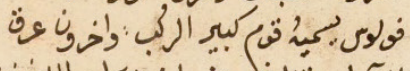

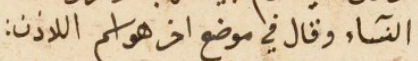

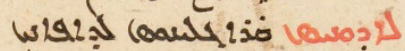
مفِنذذوبه :ورن الغار:

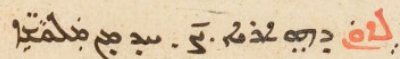

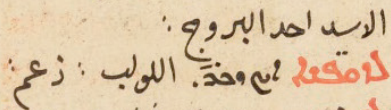
صن.

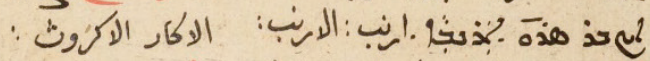

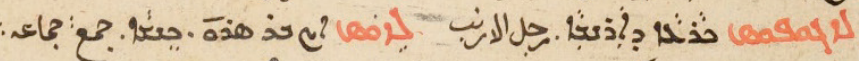

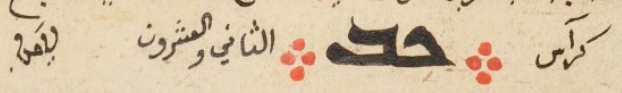

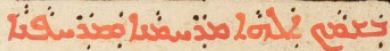
مانبز

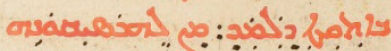

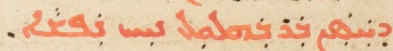

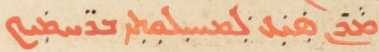
وكرن :

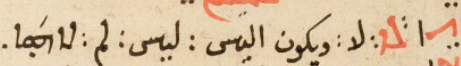

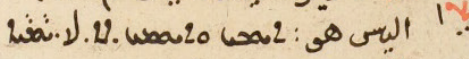

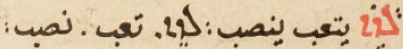

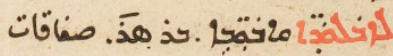

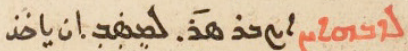

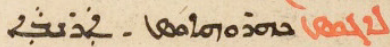
? (n)

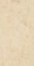
(n)

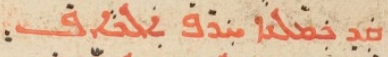
-

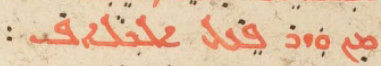
: - 7ratr - مئن 

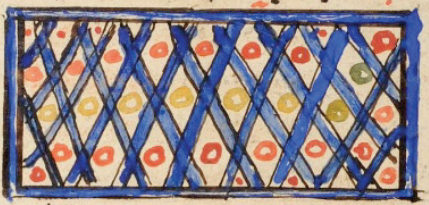




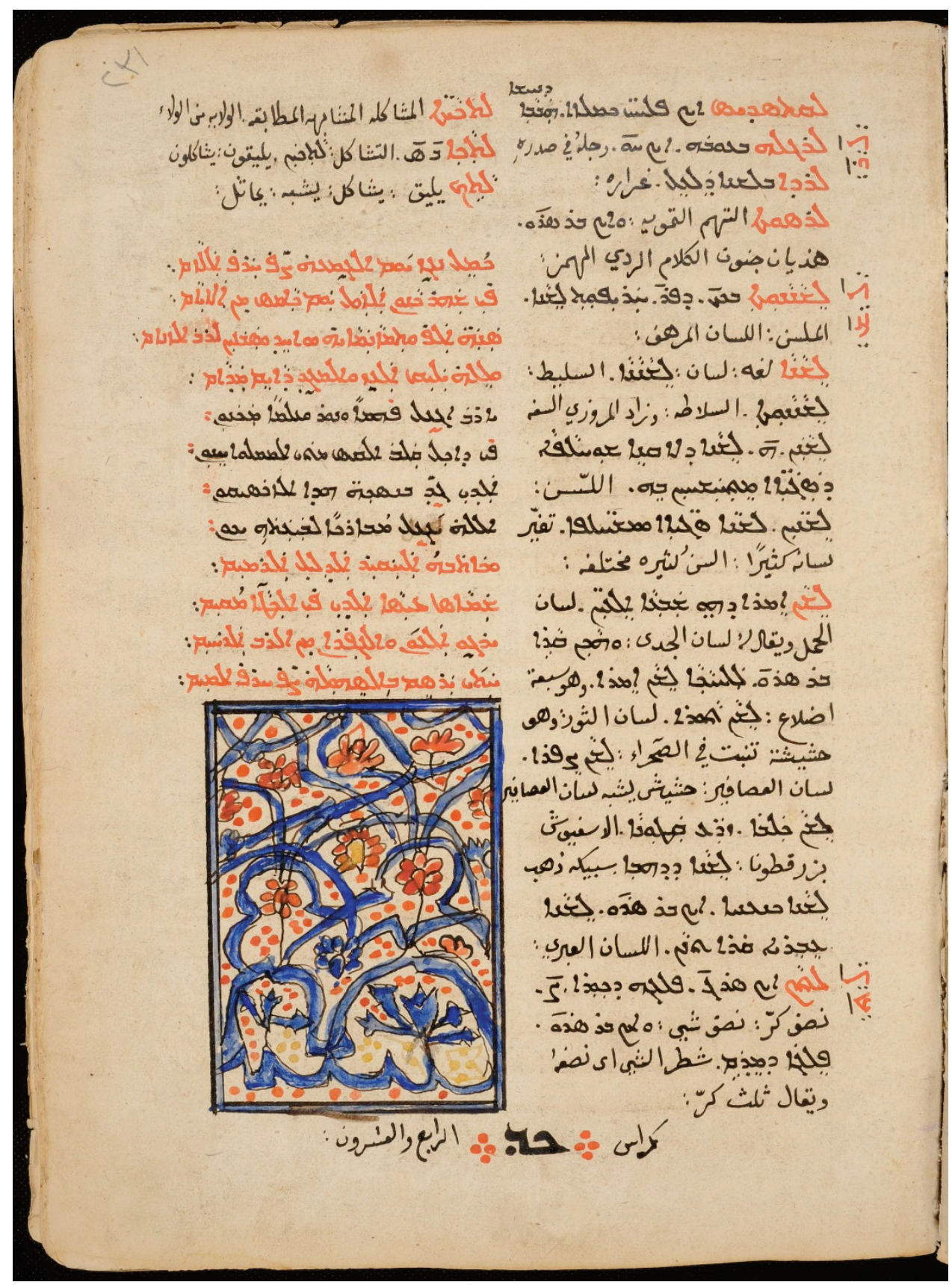

MS St. George, Alqosh; HMML DCA 84, folio 23Ir. 


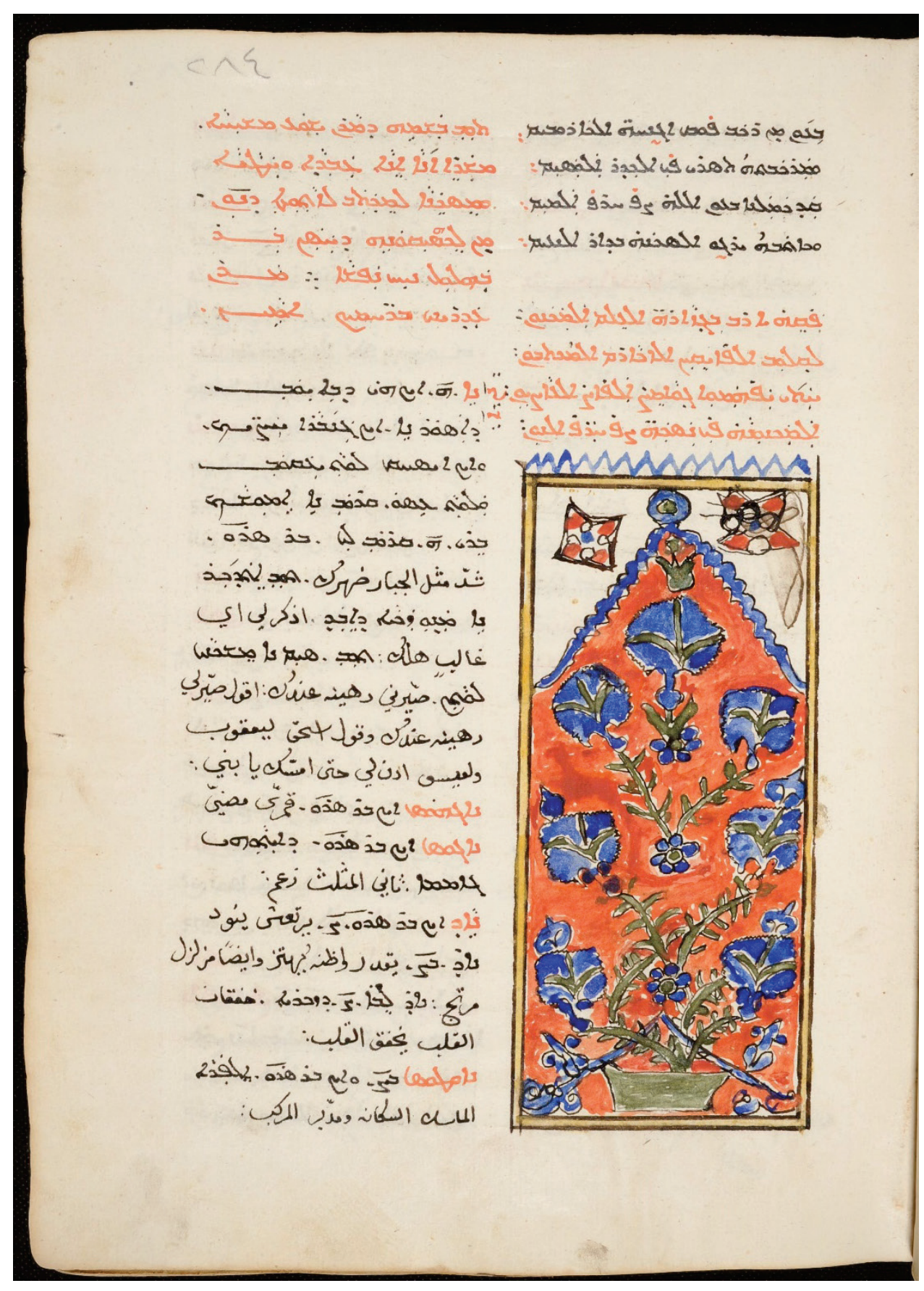

MS St. George, Alqosh; HMML DCA 84, folio 284r. 


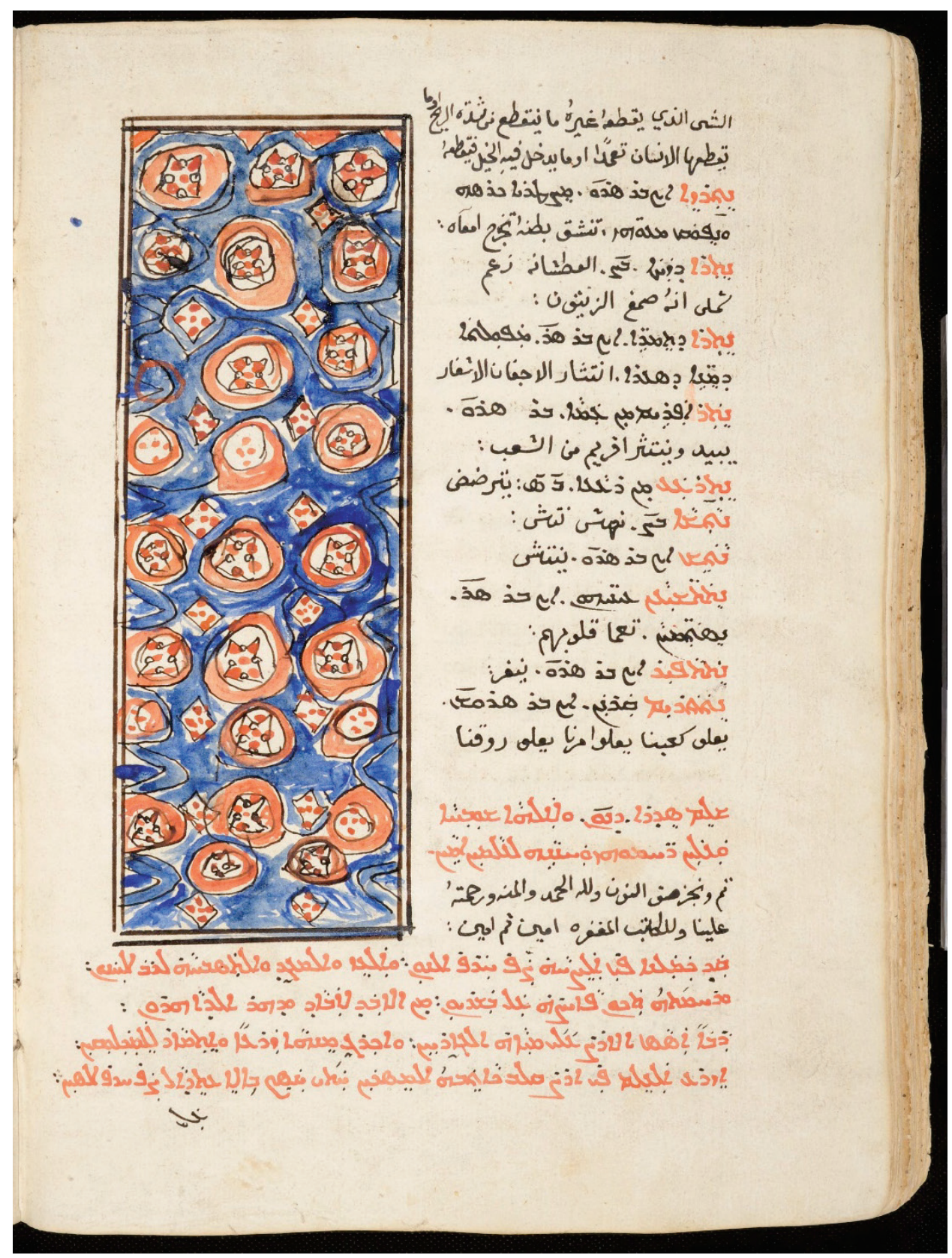

MS St. George, Alqosh; HMML DCA 84, folio 304v. 


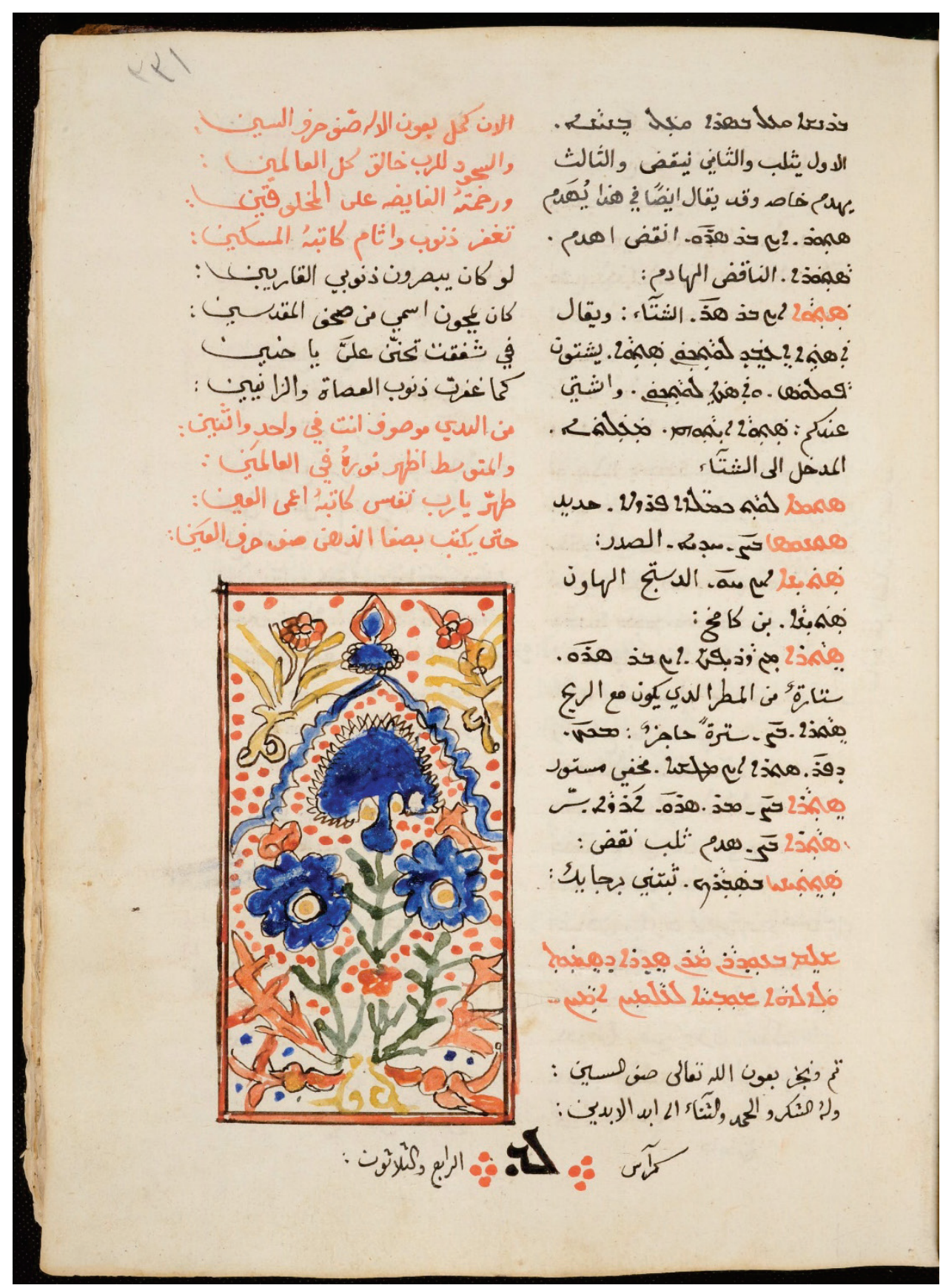

MS St. George, Alqosh; HMML DCA 84, folio 33Ir. 


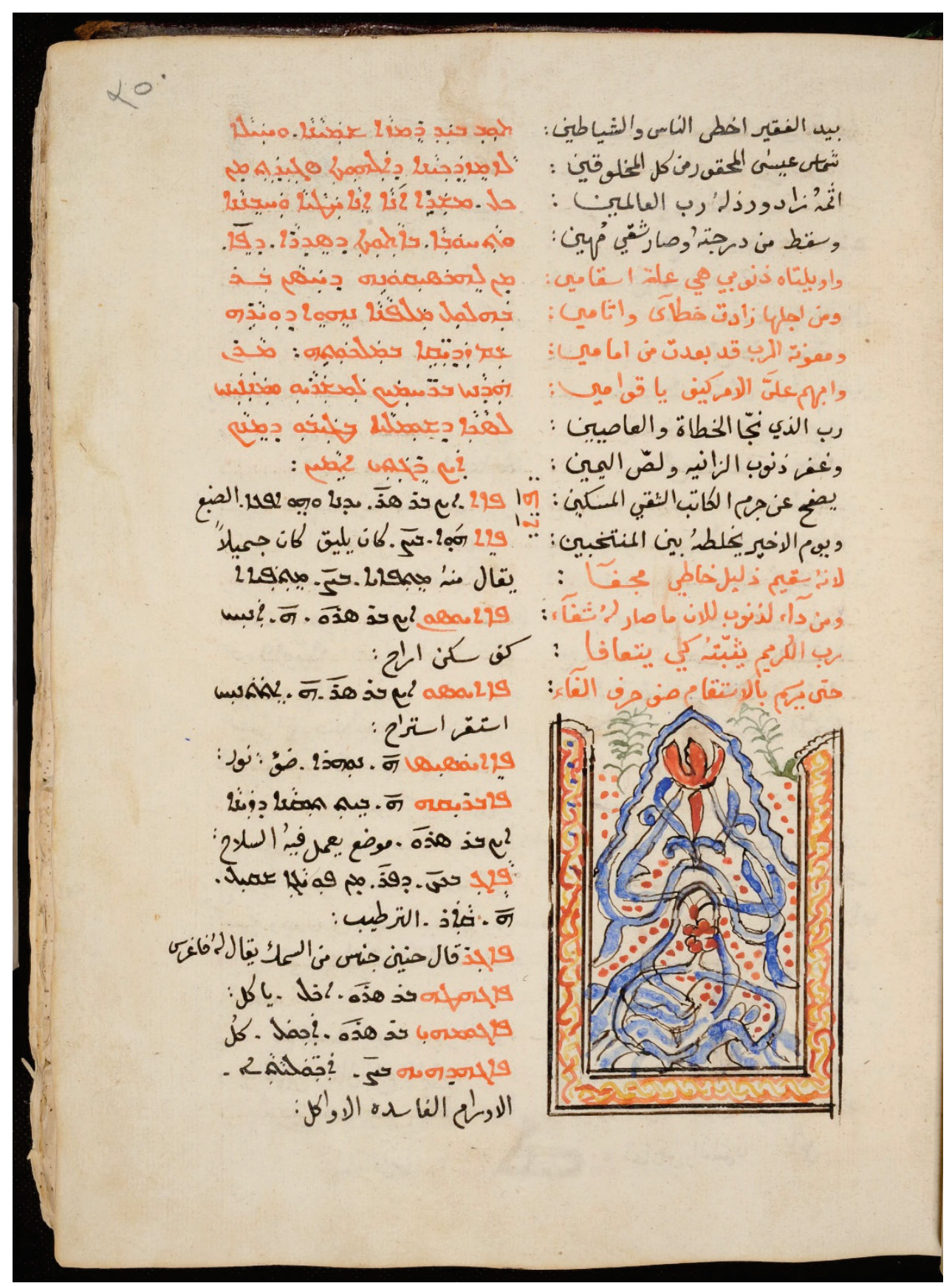

MS St. George, Alqosh; HMML DCA 84, folio 35or. 


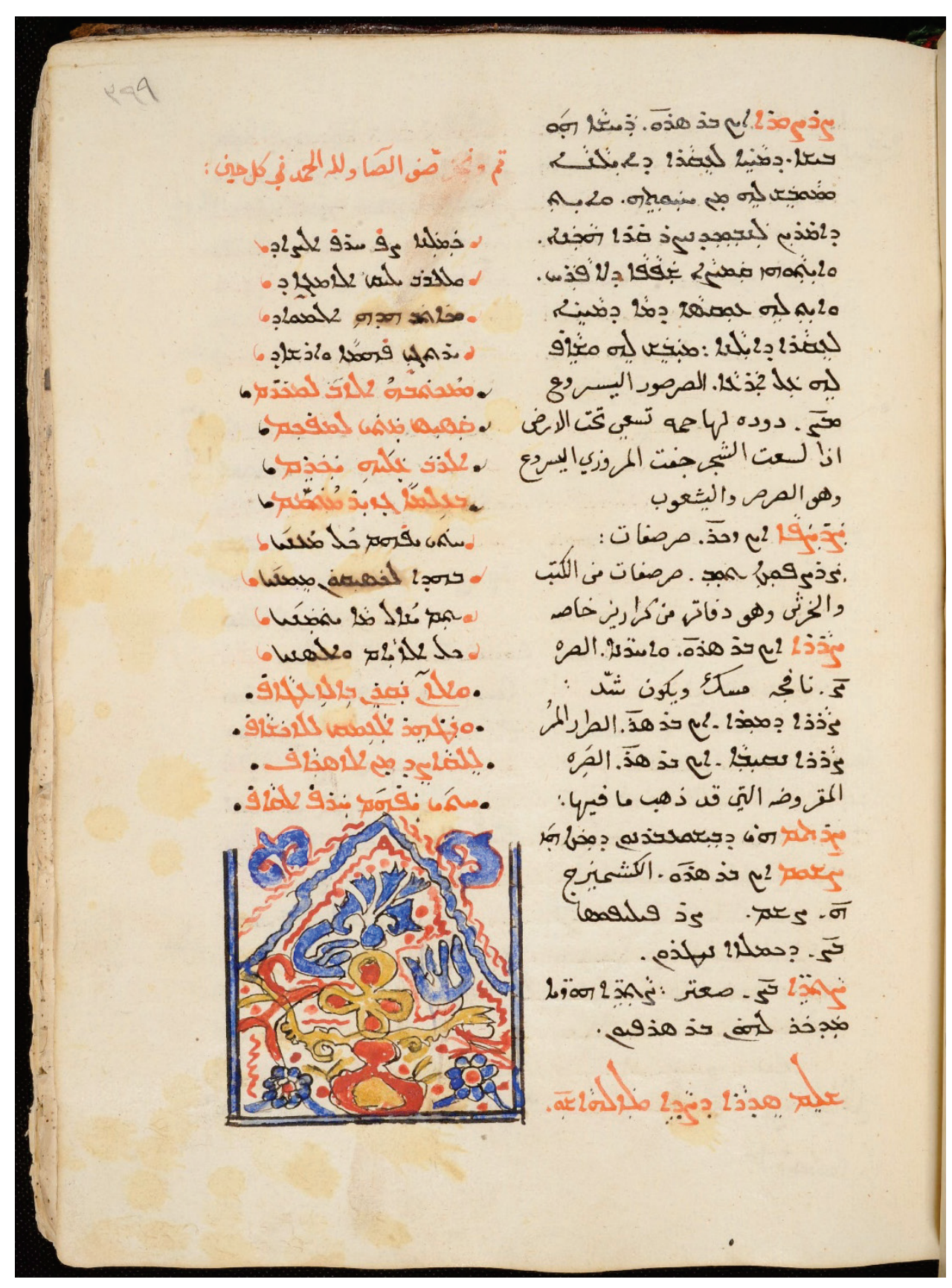

MS St. George, Alqosh; HMML DCA 84, folio 399r. 


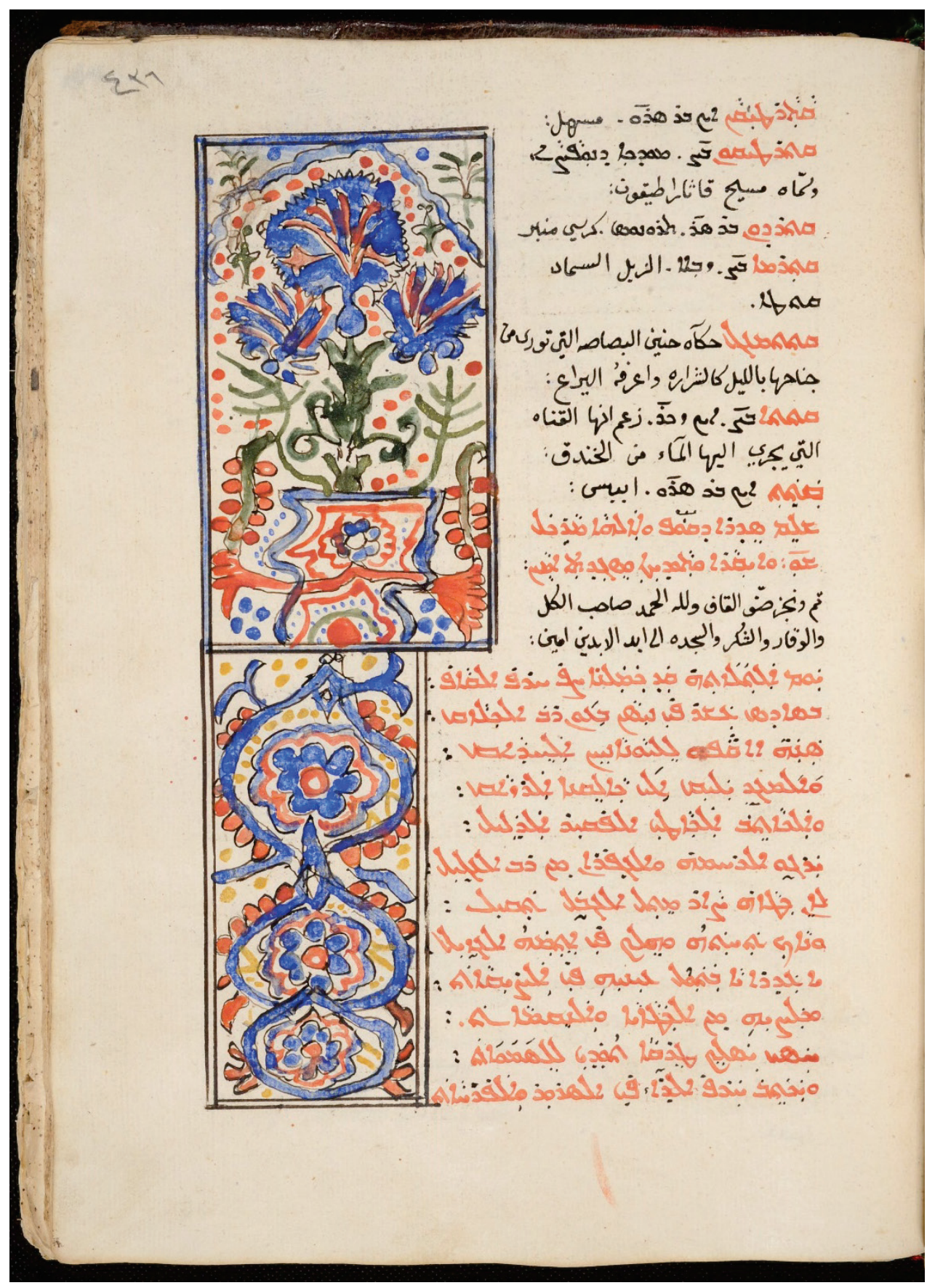

MS St. George, Alqosh; HMML DCA 84, folio 436r. 


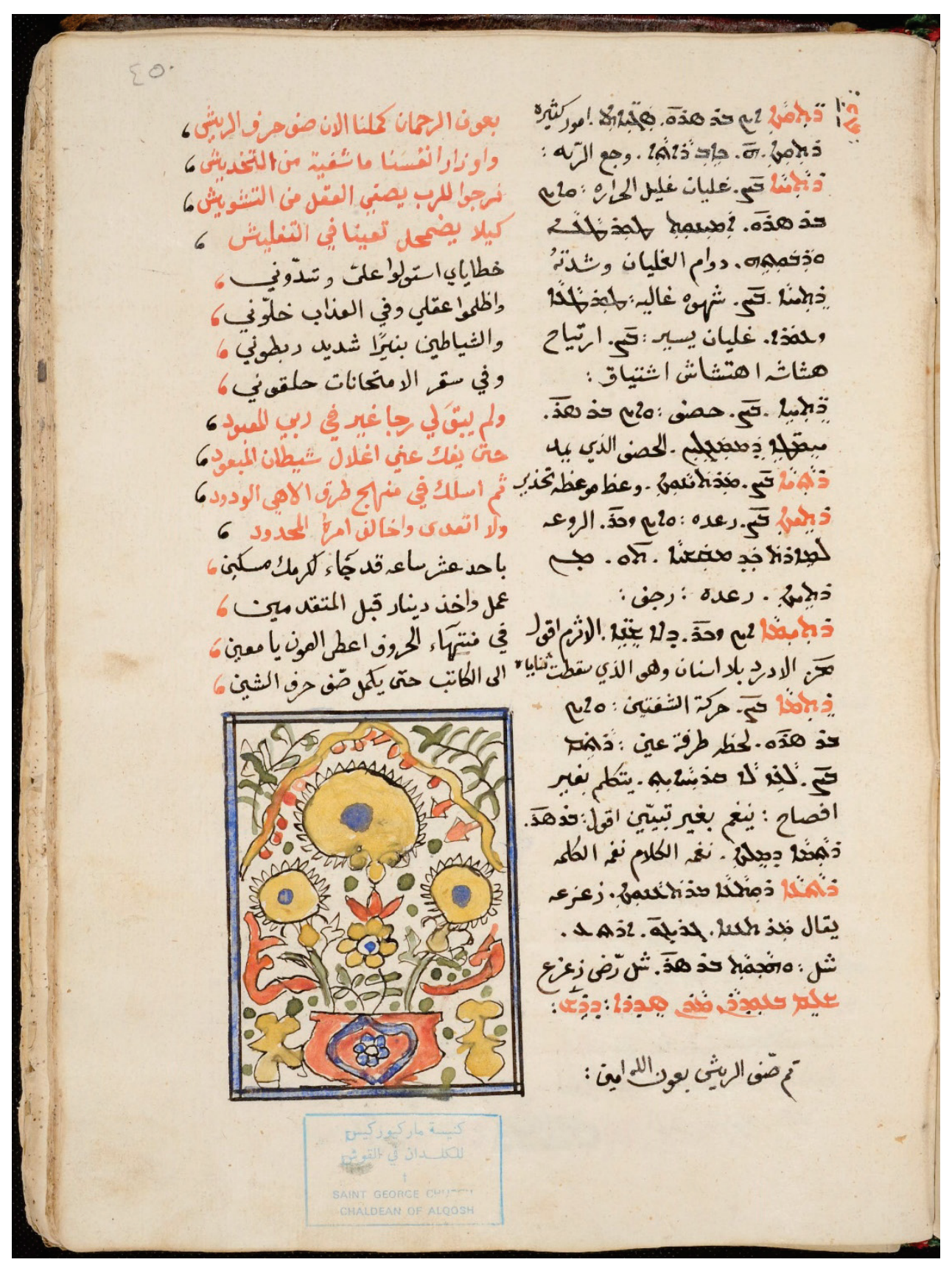

MS St. George, Alqosh; HMML DCA 84, folio 45or. 


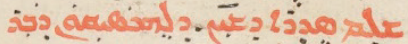
: خ

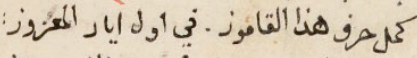

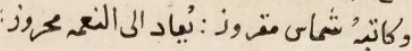

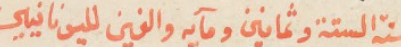

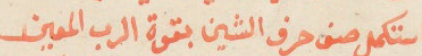

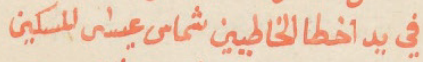

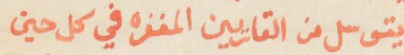

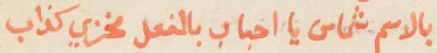

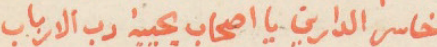

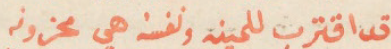

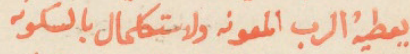

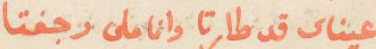

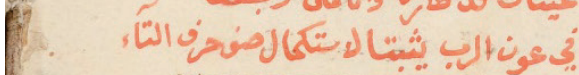

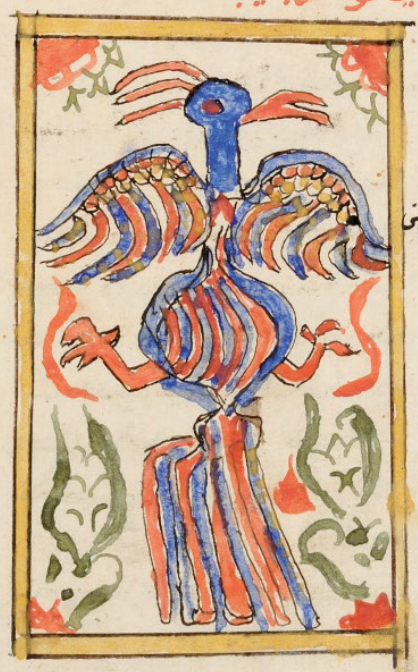

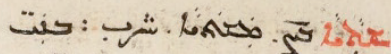

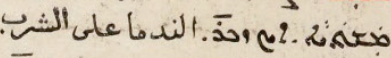

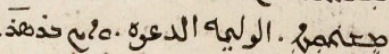
?

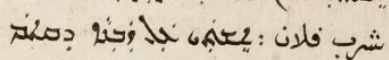

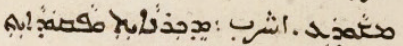

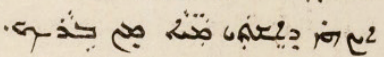
- न - م

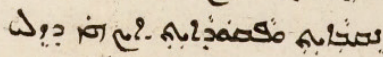

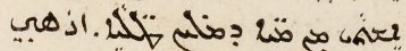

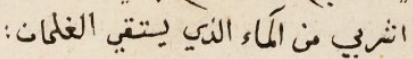

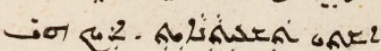

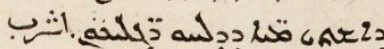

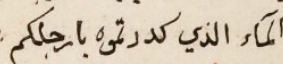

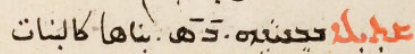
voric

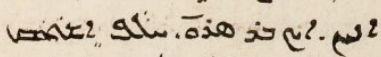

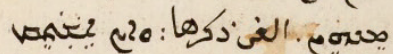

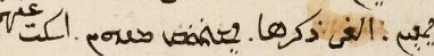

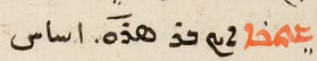

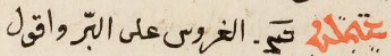

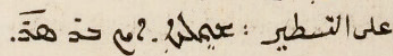

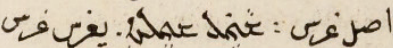

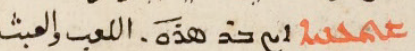
:

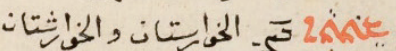
وهو أنهل والنصف والمرتب والولايه بـ

MS St. George, Alqosh; HMML DCA 84, folio 47Iv. 


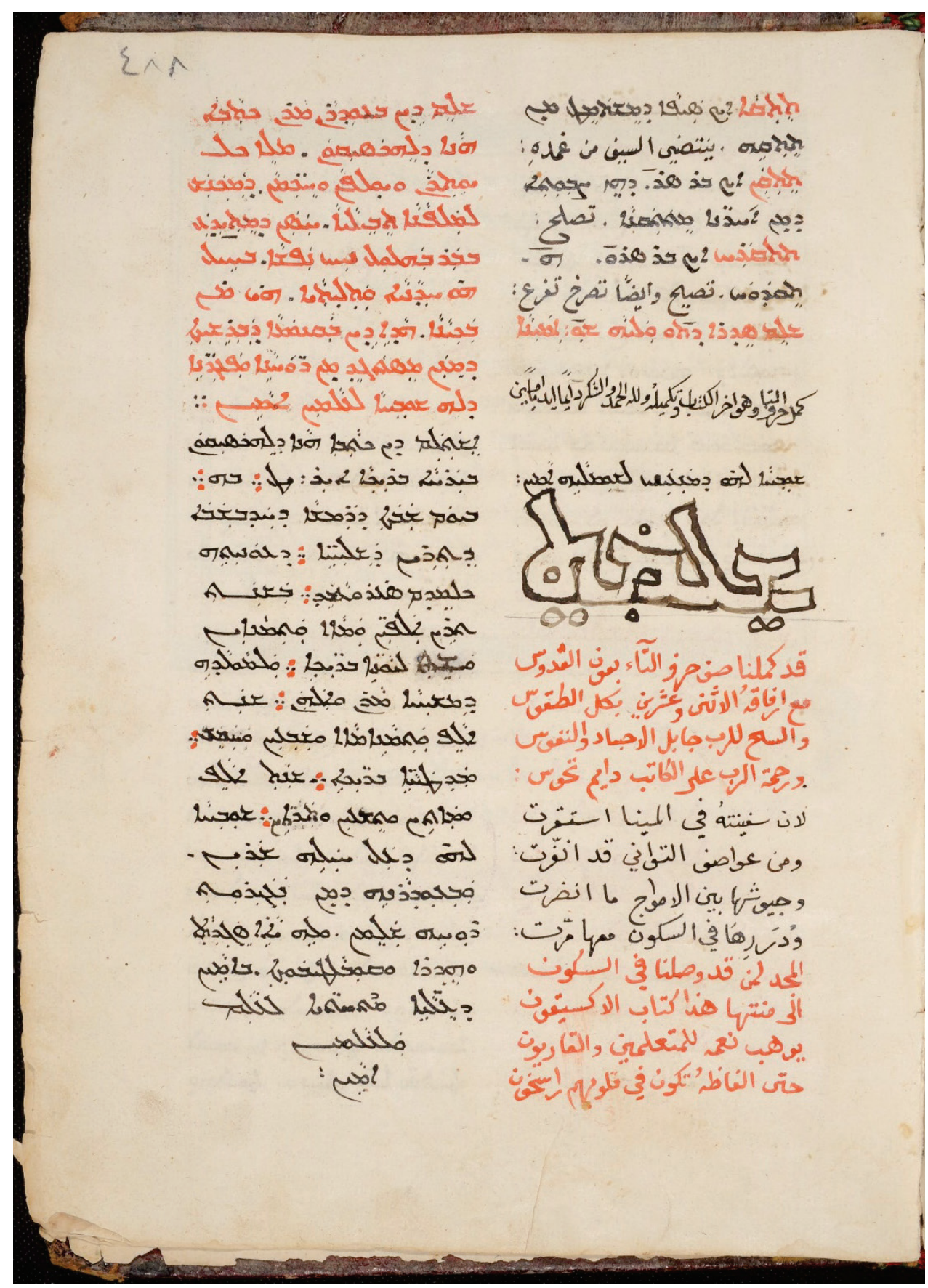

MS St. George, Alqosh; HMML DCA 84, folio 488r. 


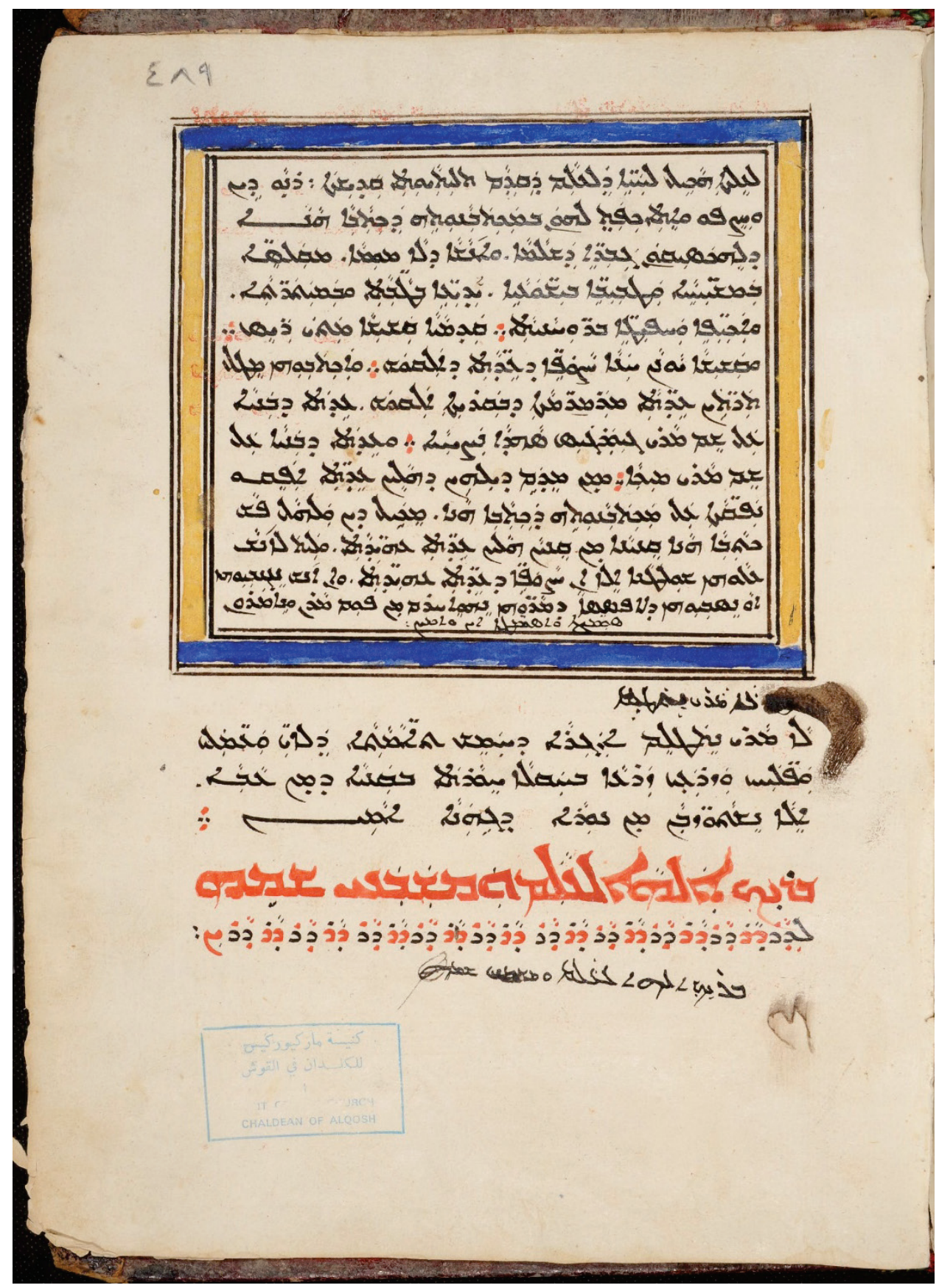

MS St. George, Alqosh; HMML DCA 84, folio 489r. 
